\title{
Management Guidelines for Pancreatic Cystic Lesions: Should we Adopt or Adapt the Current Roadmaps?
}

\author{
Filipe Vilas-Boas, Guilherme Macedo
}

Department of

Gastroenterology, Faculty of Medicine, Porto WGO Training Center - Centro Hospitalar e Universitário de São João, Porto, Portugal

Address for correspondence: Filipe Vilas-Boas, M.D. Gastroenterology Department Centro Hospitalar e

Universitário de São João Alameda Prof. Hernani Monteiro 4200-319 Porto, Portugal filipe.vboas.silva@gmail.com

\begin{abstract}
Pancreatic cystic lesions are very prevalent, especially in elderly patients and are increasingly being diagnosed because of the massive use of cross sectional imaging. Our knowledge about the natural history of these lesions is limited, especially in the case of intraductal papillary mucinous neoplasms. This fact explains why scientific societies guidelines statements are based on evidence graded as very low quality and helps the understanding of some of the different guidelines recommendations. Several guidelines have been recently revised to incorporate the new evidence published in the literature with the aim to help clinicians make the best decisions. American Gastroenterological Association guidelines, a revision of the International Consensus Guidelines, the American College of Gastroenterology and the European Study Group guidelines are the most recent.

Herein we review the current guidelines on pancreatic cysts and focus our discussion on controversies and updates about the best imaging modalities, the indications for endoscopic ultrasound guided fine needle aspiration, cyst fluid analysis, indications for resection and surveillance strategies.
\end{abstract}

Key words: pancreatic cystic lesion - IPMN- guidelines - endoscopic ultrasound - cyst fluid analysis.

Abbreviations: ACG: American College of Gastroenterology; AGA: American Gastroenterological Association; BD: branch-duct; CT: computer tomography; CE-EUS: contrast-enhanced endoscopic ultrasound; CLE: confocal laser endomicroscopy; ESG: European Study Group; EUS: endoscopic ultrasound; EUS-FNA: endoscopic ultrasound guided fine needle aspiration; HGD: high-grade dysplasia; HRF: high-risk features; HRS: high-risk stigmata; ICG: International Consensus Guidelines; IPMN: intraductal papillary mucinous neoplasm; MCN: mucinous cystic neoplasia; MD: main duct; MRCP: Magnetic Resonance Cholangiopancreatography; NGS: next generation sequencing; PCL: pancreatic cystic lesions; SCA: serous cystadenoma; WF: worrisome features.

\section{INTRODUCTION}

The diagnosis of pancreatic cystic lesions (PCLs) is increasingly performed. Two recent studies reporting on the use of cross sectional imaging for health screening programs that included around 25,000 patients showed a global prevalence of PCLs around 2.5\% $[1,2]$.

Patients with PCLs have an increased risk of pancreatic malignancy compared with the general population [3]. Using the data of a large cohort of veterans, Munigala et al. [3] reported an overall 19.64 (95\% CI, 12.12-31.82; p <0.0001) times higher risk of pancreatic cancer in these patients compared with the rest of the patients in the database.

In PCLs, malignancy occurs virtually only in those with mucinous structure. Branch duct (BD)-intraductal papillary mucinous neoplasms (IPMNs) comprise the majority of incidental pancreatic cystic lesions [4] and pose a great challenge to clinicians because they are precursor lesions of pancreatic adenocarcinoma.

However, the rate of malignancy development in IPMNs during follow-up is low. A systematic review of 37 case series predominantly from Japan and Italy reported 112 invasive cancers in 3,980 patients during 14,830 patient-years of followup. The proportion of patients developing invasive neoplasia was $2.8 \%$ overall ( $95 \% \mathrm{CI}, 1.8 \%-4.0 \%), 0.72 \%$ per year [5].

The development of trustworthy guidelines is a key priority for healthcare providers and is necessary to promote the best 
care for patients [6]. Current guidelines on PCLs are too many and confusing for the clinicians.

This paper will address the main differences between the most recently published guidelines on PCLs, in an effort to assist clinicians in the management of these patients. We will focus on the controversies and updates regarding the recommended imaging modalities, the indications for endoscopic ultrasound guided fine needle aspiration (EUSFNA), cyst fluid analysis, surgery and surveillance strategies.

\section{CURRENT GUIDELINES}

American Gastroenterological Association (AGA) guidelines [7], a revision of the International Consensus Guidelines (Fukuoka-ICG) [8], the American College of Gastroenterology (ACG) [9] and the European Study Group guidelines (ESG) [10] were recently published.

In medical science, guidelines should be evidence-based, developed as a group process using validated methods to achieve consensus after rigorous disclosure of conflicts of interest $[6,11]$. The Grading of Recommendations Assessment, Development and Evaluation (short GRADE) is a transparent approach to grading quality (or certainty) of evidence and strength of recommendations and is now considered the standard in guideline development. Except for the revised ICG (consensus symposium), the other three more recent guidelines were developed using the GRADE framework. Guidelines on PCLs are broadly concordant in that patients with main duct (MD)-IPMNs, mixed-IPMNs and solid pseudopapillary neoplasia (SPN) should be evaluated for surgery. The same is true for BD-IPMNs with "high-risk stigmata" (HRS) defined by the ICG (enhancing solid nodule or definite solid nodule $\geq 5 \mathrm{~mm}$, positive cytology for malignancy or main pancreatic duct - MPD - dilation over $10 \mathrm{~mm}$ ).

There is a consensus that the risk of malignancy in mucinous cystic neoplasia (MCN) is low if the lesion is smaller than 4 $\mathrm{cm}$ without a mural nodule. In the case of serous cystadenoma (SCA) there is no need for resection or surveillance, except if the lesion is causing symptoms.

On the other hand, especially in the case of BD-IPMNs, guidelines are discordant concerning the EUS indications, the impact of cyst size, the threshold for surgery, the surveillance intervals and the discontinuation of surveillance.

Several reasons might explain the disagreement of the different medical societies guidelines. The most obvious is our poor knowledge of PCLs natural history and the low quality of the evidence supporting the guidelines statements. In addition, the main focus used to be on cyst features rather than on the patient characteristics and ultimate prognosis.

\section{Imaging Modalities}

All current guidelines agree that magnetic resonance cholangiopancreatography (MRCP) is the best procedure to characterize the pancreatic cysts and ICG recommends its use in all patients with cysts $\geq 5 \mathrm{~mm}$ in size. Pancreatic protocol computed tomography (CT) is the alternative modality suggested by the ICG, ACG and the ESG to characterize PCLs.

The reported accuracy in identifying the specific type of PCL is between $40 \%$ and $95 \%$ for MRCP and between $40 \%$ and $81 \%$ for CT [12]. MRCP is more sensitive to detect the communication between the PCL and the pancreatic duct, the presence of mural nodules or internal septa [12]. Multifocality pointing towards the IPMN diagnosis is better evaluated by MRCP. But the presence of calcifications is better diagnosed using CT.

Sainani et al. [13] retrospectively compared the performance of dedicated pancreatic protocols CT and MRCP for 38 pathologically confirmed PCLs $\leq 3 \mathrm{~cm}$ in size. They found a better accuracy for MRCP to detect morphologic features of the cysts, but the differences were not statistically significant. More importantly, the accuracy of these two techniques for assessment of histological aggressiveness was similar (CT vs MRI, $75-78 \%$ vs 78-86\%, respectively; p > 0.05) [13].

\section{EUS-FNA}

Endoscopic Ultrasound is considered useful in the evaluation of pancreatic cysts because of its superior spatial resolution when compared with cross sectional imaging studies [14]. Endoscopic ultrasound guided-FNA with cyst fluid analysis will likely have little benefit as a first test or for the surveillance of asymptomatic solitary or multiple cysts $<1 \mathrm{~cm}$ and in the presence of classic microcystic SCA. On the contrary, it will be useful in the differential diagnosis of macrocystic SCA versus mucinous cystadenoma, in the evaluation of cystic degeneration of a solid lesion, in the diagnosis of main duct involvement in IPMNs and to confirm presence of solid component/mural nodule. Cysts that have clear indication for resection based on imaging or presence of symptoms do not need EUS.

Accuracy of EUS imaging alone in the differentiation of mucinous versus non-mucinous cysts is around 50\% [15]. Because of this limitation, EUS-guided sampling in PCLs may help in the differential diagnosis and risk stratification. The recent technical guideline of the European Society of Gastrointestinal Endoscopy (ESGE) for EUS-guided sampling recommends emptying the cyst with a single pass in the case of lesions without a solid component. When the lesion contains a solid part, this component should be targeted because samples were shown to be more accurate for diagnosis than fluid aspirates [16].

Moreover, new diagnostic modalities for the evaluation of PCLs involving EUS, such as contrast-enhanced-EUS (CEEUS) or confocal laser endomicroscopy (CLE) were recently developed and several papers report on their usefulness [15].

Contrast-enhanced-EUS is nowadays widely used as an adjunct for the differential diagnosis of pancreatic solid lesions. In the case of PCLs, Fusaroli et al. [17] demonstrated that CE-EUS allowed differentiation between pseudocysts (hypoenhancement) and cystic neoplasms (hyperenhancement) but could not differentiate between SCAs and MCNs (both with hyperenhancement). Also CE-EUS clearly showed malignant vegetations inside PCLs as solid components with hyperenhancement [17].

The revised ESG state that CE-EUS should be considered for the evaluation of mural nodules and septations [10]

Confocal laser endomicroscopy is a real-time laserassisted microscopic imaging of tissue facilitating in vivo histopathology. Its first use in pancreatic lesions was described in 2011 [18]. Four important studies (INSPECT [18], DETECT 
[19], CONTACT [20] and CONCYST-01 [21]) focused on the characterization of PCLs using CLE. A meta-analysis that included two studies that applied CLE for the diagnosis of PCLs produced a pooled sensitivity and specificity of $68 \%$ and $90 \%$, respectively [22]. The accuracy of CLE for classifying PCLs on the basis of their malignant potential is low at $46 \%$, and the inter-observer agreement for identification of CLE findings is slight $(\kappa=0.13)$ [23]. This new technique will hopefully allow better recognition of the type of lesion and risk for malignancy in the near future, but at this time, its use is not recommended outside clinical trials.

The main differences between the guidelines concerning EUS are related to the indications (Table I). The ESG suggests EUS in the presence of clinical or radiological features of concern and the ACG guideline recommends EUS in the presence of any of the following: $\mathrm{MD}>5 \mathrm{~mm}$, cyst size $\geq 3 \mathrm{~cm}$ or change in MD caliber with upstream atrophy. According to the revised ICG, EUS is indicated in the presence of any of the so called "worrisome features" (WF) and AGA guidelines suggest its use only if there are two or more positive high-risk features (HRF) (dilated $\mathrm{MD} \geq 5 \mathrm{~mm}$, cyst size $\geq 3 \mathrm{~cm}$ or nonenhancing solid component) on MRCP.

Table I. Indications for EUS-FNA

\begin{tabular}{|c|c|}
\hline Guideline & EUS-FNA \\
\hline $\begin{array}{l}\text { ICG (Revised } \\
\text { Fukuoka 2017) }\end{array}$ & ${ }^{\star}$ Worrisome features \\
\hline AGA 2015 & $* * \geq 2$ high-risk features \\
\hline European 2018 & $\begin{array}{l}\text { Clinical or radiological features of concern; } \\
\text { Hyperenhancement on CE-EUS }\end{array}$ \\
\hline ACG 2018 & $\begin{array}{l}\text { Cysts in which the diagnosis is unclear, and where } \\
\text { the results are likely to alter management }\end{array}$ \\
\hline
\end{tabular}

ACG: American College of Gastroenterology; AGA: American Gastroenterological Association; ICG: International Consensus Guidelines; ${ }^{*}$ Worrisome features: pancreatitis, cyst $\geq 3 \mathrm{~cm}$, enhancing mural nodule $<5$ $\mathrm{mm}$, thickened/enhancing cyst walls, main duct size 5-9 mm, abrupt change in caliber of pancreatic duct with distal pancreatic atrophy, lymphadenopathy, increased serum CA19-9, cyst growth rate $\geq 5 \mathrm{~mm} / 2$ years; ${ }^{*}$ AGA Highrisk features: dilated MPD ( $\geq 5 \mathrm{~mm}), \geq 3 \mathrm{~cm}$ cyst or non-enhancing solid component

The previous ICG published in 2012 defined WF as presence of pancreatitis, cyst size $\geq 3 \mathrm{~cm}$, thickened/enhancing cyst walls, MPD size 5-9 $\mathrm{mm}$ in diameter, abrupt change in the caliber of the pancreatic duct with distal pancreatic atrophy and lymphadenopathy [24]. In the revision of the ICG published in 2017 the authors added the presence of enhancing mural nodule $<5 \mathrm{~mm}$, increased serum CA19-9 and cyst growth rate $\geq 5 \mathrm{~mm} / 2$ years as WF [8].

The requirement of two or more positive HRF to perform EUS as recommended by the AGA guideline has been questioned. Kohli et al. [25] published a retrospective cohort study that included 210 patients who had EUS for PCLs characterization between 2004 and 2015. The requirement of $\geq 2 \mathrm{HRF}$, based on AGA practice guidelines, would have decreased the number of EUS procedures by $91 \%$, but reduced the sensitivity for pancreatic malignancy to $50 \%$ [25].

\section{Cyst Fluid Analysis}

A CEA level of $192 \mathrm{ng} / \mathrm{mL}$ was found to be optimal by using receiver operating characteristic curves, with a $75 \%$ sensitivity and $84 \%$ specificity for differentiating between mucinous and non-mucin producing cysts, but CEA levels are inaccurate to differentiate benign versus malignant mucinous PCLs [26].

The combination of clinical and molecular features was recently pointed to be more accurate for assessing the cyst type and the need for resection.

Springer et al. [27] reported on the use of massive parallel DNA sequencing in 130 cyst fluid samples collected at the time of EUS or from resected surgical specimens in an interval of 9 years. The composite molecular markers (presence of a mutation in SMAD4, chromosome $17 \mathrm{LOH}$ - region containing RNF43 - or aneuploidy in chromosome 5p, 8p, 13q, or 18q) correctly identified IPMNs with high-grade dysplasia or invasive carcinoma with a sensitivity of $75 \%$ and a specificity of $92 \%$. The combination of both clinical and molecular features increased the sensitivity to $89 \%$, but the specificity was only $69 \%$ [27].

Singhi et al. [28] prospectively evaluated the use of the next generation sequencing (NGS) using cyst fluid obtained during EUS-FNA (626 specimens from 595 patients). The assay targeted several genes known to be mutated and/or deleted in PCLs (KRAS, GNAS, NRAS, HRAS, BRAF, CTNNB1, TP53, PIK3CA, PTEN and AKT1). The authors found that mutations in KRAS and GNAS are highly sensitive and specific for IPMN, but not MCN. Moreover, detection of mutations/deletions in TP53, PIK3CA and/or PTEN were highly sensitive and specific for IPMNs with advanced neoplasia [28].

Besides the measurement of tumor markers and molecular studies, cyst fluid analysis allows cytologic evaluation that may distinguish different types of cysts (serous vs. mucinous) and may permit the grading of the epithelium of mucinous cysts [15]. But the cytological interpretation is limited by specimen cellularity, degeneration, and contamination with gastrointestinal epithelium. Concerning cytology, a metaanalysis published in 2013 revealed 42\% sensitivity and 99\% specificity for differentiating mucinous versus non-mucinous PCLs [29].

All guidelines on PCLs beside ICG recommend cyst fluid analysis with CEA level determination and cytology. The revision of ICG still considers EUS-FNA for cytology as investigational but mentions the added value of cyst fluid CEA levels. Most guidelines consider molecular analysis as investigational and not ready for clinical practice. The ACG guideline suggests that the use of molecular markers may be considered in the case of indeterminate diagnosis of cyst type when the results are likely to change the management (Table II).

In fact, molecular studies are nowadays still considered costly and may not add to the standard analysis.

\section{Surgery}

In a retrospective series from Massachusetts General Hospital involving 851 individuals undergoing resection of PCLs over 33 years, $60 \%$ of the lesions had a risk of harboring malignancy or progress to malignancy (about 40\% IPMNs; 
Table II. Cyst fluid analysis

\begin{tabular}{lcccc}
\hline Guideline & CEA & Biochemistry & Cytology & Molecular analysis \\
\hline ICG (Revised Fukuoka 2017) & $\mathrm{M}$ & Amylase & Investigational & KRAS/GNAS \\
& & & & Investigational \\
AGA 2015 & $\mathrm{R}$ & $(-)$ & $\mathrm{R}$ & Investigational \\
European 2018 & $\mathrm{R}$ & Lipase & $\mathrm{R}$ & $\begin{array}{c}\text { KRAS/GNAS } \\
\text { (conditional) }\end{array}$ \\
ACG 2018 & $\mathrm{R}$ & $(-)$ & $\mathrm{R}$ & Not ready for clinical practice
\end{tabular}

ACG: American College of Gastroenterology; AGA: American Gastroenterological Association; ICG: International Consensus Guidelines; CEA: Carcinoembryonic antigen; GNAS: adenylate cyclase-stimulating G alpha protein; KRAS: Kirsten rat sarcoma viral oncogene homolog; M: mentioned; R: recommended; (-): not mentioned.

$20 \% \mathrm{MCN} ; 3 \% \mathrm{SPN}$ ). This series also shows that the most incidentally found PCLs are IPMNs [30].

IPMNs encompass a spectrum of lesions from adenoma to invasive carcinoma, and are considered precancerous lesions [31].

The mean frequency of invasive carcinoma and high-grade dysplasia (HGD) in resected BD-IPMN is $31.1 \%$ and that of invasive cancer is $18.5 \%$ [8].

Pancreatic surgery carries a significant risk of morbidity, even in high-volume centers. Valsangkar et al. [30] found a 38\% postoperative complication rate for PCLs resection. Crippa et al. [31] reported mortality rates of $1-3 \%$ and morbidity rates of $30-60 \%$ after surgical resection of IPMNs, depending on resection types.

Surgery in IPMNs is most valuable in the case of lesions harboring HGD or carcinoma in situ, so the focus should be to refine the diagnosis of HGD.

Several features in mucinous lesions are associated with an increased risk for HGD or cancer and are used to determine indications for resection. Table III shows the indications for surgery in PCLs according to society guidelines.

All guidelines (except AGA guidelines) recommend cyst resection in the presence of jaundice or acute pancreatitis or in the presence of positive cytology for malignancy.

Concerning the cyst size, the ICG and AGA moved away from size alone as criterion to indicate surgery because of its poor predictive value for invasive carcinoma and HGD, but the revised ESG consider lesion size $\geq 4 \mathrm{~cm}$ as a relative indication for resection. ACG recommends that for mucinous cysts $\geq 3$ $\mathrm{cm}$ the pros and cons of surgery versus surveillance should be discussed.
Recent data showed that the growth rate may be more important than the cyst size itself. A large retrospective multicentric study from the USA and China [32] including 284 patients with BD-IPMNs without WF or HRS, showed that malignant BD-IPMNs (18.6 vs. $0.8 \mathrm{~mm} /$ year; $\mathrm{p}=0.05$ ) grew at a faster rate compared to benign BD-IPMNs and that a growth rate $\geq 5 \mathrm{~mm} /$ year had a hazard ratio of 19.5 (95\% CI 2.4-157.8) for malignancy.

The presence of mural nodules was found to have the highest diagnostic odds ratio for malignancy in BD-IPMNs in a meta-analysis of 23 articles that included 1373 patients [33]. In fact, in all guidelines, mural nodules are an absolute indication for resection. The cut-off value size of mural nodules to identify high-risk lesions is set at $5 \mathrm{~mm}$ by the revised ICG and the ESG. A mural nodule $\geq 5 \mathrm{~mm}$ on EUS has a sensitivity of $73-85 \%$ and specificity of $71-100 \%$ for HGD or cancer [10].

Concerning MD, studies showed that the risk of advanced histology (HGD or cancer) was correlated with the duct size and with the presence of abrupt caliber change. In the previously cited meta-analysis [33], Kim et al. found the MD dilation to have a diagnostic odds ratio of 3.4 (95\% CI 2.3-5.2), the highest after mural nodules. This finding justifies the inclusion of MD dilation by all the recent guidelines as an important feature for the management of IPMNs. An MD $\geq 10 \mathrm{~mm}$ is considered an absolute indication for surgery by the ICG and the ESG, and a duct size of $5-9.9 \mathrm{~mm}$ is considered a WF by ICG and is a relative indication for surgery as determined by the ESG.

Of note is the recent inclusion of high serum CA 19-9 as an important predictor of advanced histology in IPMNs. Concerning the indication for resection, the revision of ICG

Table III. Indications for surgery

\begin{tabular}{|c|c|c|c|c|c|c|}
\hline Guideline & Symptoms & MPD & Mural nodule & $\begin{array}{l}\text { Positive } \\
\text { cytology }\end{array}$ & Size & Comments \\
\hline $\begin{array}{l}\text { ICG (Revised } \\
\text { Fukuoka 2017) }\end{array}$ & $\stackrel{+}{+}$ & $\geq 10 \mathrm{~mm}$ & $\stackrel{+}{+} \underset{5 \mathrm{~mm} \text { cut-off }}{+}$ & + & $-{ }^{*}$ & $\begin{array}{l}\text { HRS Consider life expectancy, } \\
\text { comorbidities and location }\end{array}$ \\
\hline AGA 2015 & NA & dilated & + & + & - & and/or 2 features \\
\hline European 2018 & $\begin{array}{l}+ \text { jaundice, } \\
\text { acute pancreatitis }\end{array}$ & $\begin{array}{c}\geq 10 \mathrm{~mm} \\
\text { (5-9.9 mm relative } \\
\text { indication) }\end{array}$ & $\stackrel{+}{+} \underset{5 \mathrm{~mm} \text { cut-off }}{+}$ & + & $\begin{array}{l}\quad \geq 4 \mathrm{~cm} \\
\text { (relative } \\
\text { indication) }\end{array}$ & $\begin{array}{l}\text { Growth rate } \geq 5 \mathrm{~mm} / \text { year } \\
\text { new-onset DM high CA19.9 }\end{array}$ \\
\hline ACG 2018 & $\begin{array}{l}+ \text { jaundice, acute } \\
\text { pancreatitis }\end{array}$ & $\geq 5 \mathrm{~mm}$ & + & + & $\geq 3 \mathrm{~cm}$ & $\begin{array}{l}\text { Growth rate } \geq 3 \mathrm{~mm} / \text { year } \\
\text { new-onset DM high CA19.9 }\end{array}$ \\
\hline
\end{tabular}

ACG: American College of Gastroenterology; AGA: American Gastroenterological Association; CA 19.9: carbohydrate antigen 19.9; DM: Diabetes Mellitus; HRS: high-risk stigmata; ICG: International Consensus Guidelines; MPD: Main pancreatic duct; NA: not applicable; ${ }^{*}$ cyst size alone is not an appropriate parameter to indicate surgery. Presence of more than one risk factor increases probability of HGD/inv carcinoma 
included high serum CA 19-9 as a new WF and the ESG states high CA $19-9$ as a relative indication for surgery.

All this discussion is focused on cyst features, but the trend is to adopt a more "patient"-centered strategy. This shift happens because of a better understanding of the natural history of PCLs, namely IPMNs.

Nowadays there is a need to personalize the decision and to focus on patient condition/status (comorbidities and expected survival) because factors besides cyst features have a significant impact on patient outcomes, and every multidisciplinary team taking care of these patients should be aware of this fact.

Two important studies brought new information concerning this problem. The first is a multicentric retrospective study from Italy and the USA that included 281 elderly patients with IPMNs (231 harboring WF and 50 HRS, as per ICG that undergone non-operative management with a median followup of 51 months) [34]. The disease-specific survival (DSS) of patients with IPMNs with WF was as high as 96\%, highlighting noncancerous mortality of these elderly patients on long-term follow-up. In the case of patients with IPMNs harboring HRS, the authors found a $40 \%$ risk of IPMN-related death reinforcing that surgical resection should be offered to fit patients [34].

The second important report about competing risks for mortality in patients with PCLs was recently published by Kwok et al. [35]. The authors included 1800 patients with PCLs stratified based on Charlson comorbidity index. There were 402 deaths during a median follow-up of 5.7 years. Only 43 deaths were PCL-related, reinforcing that the association of patient-related factors and cyst features help guide the management.

The ESG now propose the management based on cyst features but also on the patient's life expectancy and comorbidities and cyst location. This stratification allows patients to be divided into two resection strategies: preemptive surgery in the case of the presence of relative indications for surgery, and cancer surgery in the case of absolute indication(s).

\section{Surveillance}

All guidelines support surveillance when patients are not submitted to surgery (Table IV).

The main controversy and perhaps the most striking feature introduced by the 2015 AGA guidelines is the possibility of stopping surveillance.
A retrospective multicentric study from Italy including 144 IPMNs with neither WF nor HRS reveals new-onset of WF and HRS in 26 patients (18\%) after a median follow-up of 71 and 77.5 months, respectively. One out of six patients developed WF or HRS beyond 5 years of surveillance, so the authors conclude that persistent surveillance is required [36].

The possibility of stopping surveillance was evaluated by the group of Massachusetts General Hospital in a retrospective study of 577 patients with BD-IPMN, of whom 363 underwent surveillance over 5 years. Forty-five patients developed malignancy, 5 of them after 10 years of follow-up [37]. These results do not support AGA's recommendation for stopping surveillance.

The authors found, however, that cysts which remain smaller than $15 \mathrm{~mm}$ for more than 5 years might be considered low-risk. In this group of patients, the decision to stop surveillance may be adequate [37].

\section{COMMENTS AND CONCLUSION}

A solitary PCL may remain a diagnostic challenge after completion of all currently available investigations. The morphology and location of the cyst along with the presence of communication with MD and the patient characteristics, including age and gender are useful in the differential diagnosis of PCLs. In addition, a history of acute or chronic pancreatitis may point to the diagnosis of a pseudocyst (Table V). Currently, the CEA level in cyst fluid is the best modality to differentiate mucinous and non-mucin producing cysts but it is not a predictor of malignancy or dysplasia. In the future, if the usefulness of molecular studies is confirmed, analysis of key gene mutations may be part of clinical practice and aid in risk stratification.

To infer advanced histology/malignancy, the presence of mural nodules/solid component, dilation of MD and growth rate have the highest predictive value.

Several scientific societies issued guidelines for the management of PCLs. In the era of Evidence-based Medicine, clinicians must support their decisions on the best available data in the literature, but there are some controversies stated in the different guidelines that can lead to confusion. The clinicians frequently do not know which guideline they should follow. The controversies are justified by the fact that

Table IV. Surveillance (mucinous cysts)

\begin{tabular}{|c|c|c|}
\hline Guideline & Follow-up indications & Stop surveillance \\
\hline $\begin{array}{l}\text { ICG (Revised Fukuoka } \\
\text { 2017) }\end{array}$ & $\begin{array}{l}<1 \mathrm{~cm}-\mathrm{CT} / \mathrm{MRI} \text { in } 2-3 \text { years } \\
1-2 \mathrm{~cm}-\mathrm{CT} / \mathrm{MRI} \text { yearly } \mathrm{x} 2 \text { then lengthen as appropriate } \\
{ }^{\star} 2-3 \mathrm{~cm}-\text { EUS in } 3-6 \text { months then lengthen as appropriate } \\
{ }^{*}>3 \mathrm{~cm}-\mathrm{MRI} / \text { EUS every } 3-6 \text { months up to } 1 \text { year }\end{array}$ & $\begin{array}{l}\text { Lifelong } \\
\text { (until not fit for surgery) }\end{array}$ \\
\hline AGA 2015 & MRI after one year then MRI every 2 years & Stable appearance after 5 years \\
\hline European 2018 & $\begin{array}{l}\text { EUS/MRI and CA 19-9 after six months then EUS/MRI } \\
\text { and CA 19-9 yearly }\end{array}$ & $\begin{array}{l}\text { Lifelong } \\
\text { (until not fit for surgery) Intensification after } 5 \text { yrs? }\end{array}$ \\
\hline ACG 2018 & Cyst size guides surveillance (similar to ICG) & $\begin{array}{l}\text { When not fit for surgery, assess utility in those }>75 \\
\text { years }\end{array}$ \\
\hline
\end{tabular}


Table V. Features of common pancreatic cystic lesions

\begin{tabular}{|c|c|c|c|c|c|}
\hline & SCA & MCN & IPMN & SPN & PC \\
\hline Age (years) & $60-80$ & $30-50$ & $60-80$ & $20-30$ & Variable \\
\hline Gender & Female $>$ Male & Nearly all female & Male>Female & Female & Male $=$ Female \\
\hline Location & Any & Body/tail & Head & Body/tail & Any \\
\hline Malignant potential & Very rare & Moderate/high & $\begin{array}{l}\text { MD, MT - high; } \\
\text { BD - Low }\end{array}$ & Low & none \\
\hline $\begin{array}{l}\text { Communication } \\
\text { with main duct }\end{array}$ & No & No & Yes & No & Variable \\
\hline Cytology & Cuboidal cells & Columnar cells & Columnar cells & $\begin{array}{c}\text { Fibrovascular } \\
\text { stroma }\end{array}$ & Cyst contents \\
\hline
\end{tabular}

BD: branch-duct; IPMN: Intraductal papillary mucinous neoplasia; MCN: Mucinous cystic neoplasm; MD: main duct, MT - mixed type; PC: Pseudocyst; SCA: Serous cystadenomas; SPN: Solid pseudopapillary neoplasm.

data is limited, especially in regard to the natural history of BD-IPMN. All the evidence related to the management of pancreatic cysts is graded as very low quality. Furthermore, the guidelines reflect the motivations and are biased by the authors' background.

All guidelines agree on the importance of a detailed history and on the importance of MRCP for initial evaluation. EUS should be performed in the presence of any known WF. Regarding treatment and surveillance, there is consensus on the need for upfront multidisciplinary discussion and the need to include patients in the decision. Furthermore, surgery should be performed only at high-volume centers to ensure the best results with less morbidity.

The decision to observe versus to resect often remains individual. We must consider patient status, namely comorbidities and life expectancy, because recent data has evidenced that most patients will die from causes not related with a cyst.

The strict adherence to a particular guideline is probably not the best option; therefore, we should adapt a strategy aiming for a personalized approach.

Conflicts of interest: None to declare.

Authors' contribution: F.V.B. wrote the original draft of the manuscript. G.M. critically revised the manuscript and approved the final version.

\section{REFERENCES}

1. de Jong K, Nio CY, Hermans JJ, et al. High prevalence of pancreatic cysts detected by screening magnetic resonance imaging examinations. Clin Gastroenterol Hepatol 2010;8:806-811. doi:10.1016/j.cgh.2010.05.017

2. Chang YR, Park JK, Jang JY, Kwon W, Yoon JH, Kim SW. Incidental pancreatic cystic neoplasms in an asymptomatic healthy population of 21,745 individuals: Large-scale, single-center cohort study. Medicine (Baltimore) 2016;95:e5535. doi:10.1097/MD.0000000000005535

3. Munigala S, Gelrud A, Agarwal B. Risk of pancreatic cancer in patients with pancreatic cyst. Gastrointest Endosc 2016;84:81-86. doi:10.1016/j. gie.2015.10.030

4. Shimizu Y, Yamaue H, Maguchi H, et al. Predictors of malignancy in intraductal papillary mucinous neoplasm of the pancreas: analysis of
310 pancreatic resection patients at multiple high-volume centers. Pancreas 2013;42:883-888. doi:10.1097/MPA.0b013e31827a7b84

5. Scheiman JM, Hwang JH, Moayyedi P. American gastroenterological association technical review on the diagnosis and management of asymptomatic neoplastic pancreatic cysts. Gastroenterology 2015;148:824-848.e22. doi:10.1053/j.gastro.2015.01.014

6. Cooke CR, Gould MK. Advancing clinical practice and policy through guidelines: the role of the American Thoracic Society. Am J Respir Crit Care Med 2013;187:910-914. doi:10.1164/rccm.201301-0009PP

7. Vege SS, Ziring B, Jain R, Moayyedi P; Clinical Guidelines Committee; American Gastroenterology Association. American gastroenterological association institute guideline on the diagnosis and management of asymptomatic neoplastic pancreatic cysts. Gastroenterology 2015;148:819-822. doi:10.1053/j.gastro.2015.01.015

8. Tanaka M, Fernandez-Del Castillo C, Kamisawa T, et al. Revisions of international consensus Fukuoka guidelines for the management of IPMN of the pancreas. Pancreatology 2017;17:738-753. doi:10.1016/j. pan.2017.07.007

9. Elta GH, Enestvedt BK, Sauer BG, Lennon AM. ACG Clinical Guideline: Diagnosis and Management of Pancreatic Cysts. Am J Gastroenterol 2018;113:464-479. doi:10.1038/ajg.2018.14

10. European Study Group on Cystic Tumours of the Pancreas. European evidence-based guidelines on pancreatic cystic neoplasms. Gut 2018;67:789-804. doi:10.1136/gutjnl-2018-316027

11. Vakil N. Editorial: consensus guidelines: method or madness? Am J Gastroenterol 2011;106:225-227. doi:10.1038/ajg.2010.504

12. Jang DK, Song BJ, Ryu JK, et al. Preoperative Diagnosis of Pancreatic Cystic Lesions: The Accuracy of Endoscopic Ultrasound and CrossSectional Imaging. Pancreas 2015;44:1329-1333. doi:10.1097/ MPA.0000000000000396

13. Sainani NI, Saokar A, Deshpande V, Fernandez-del Castillo C, Hahn P, Sahani DV. Comparative performance of MDCT and MRI with MR cholangiopancreatography in characterizing small pancreatic cysts. AJR Am J Roentgenol 2009;193:722-731. doi:10.2214/AJR.08.1253

14. Ahmad NA, Kochman ML, Brensinger C, et al. Interobserver agreement among endosonographers for the diagnosis of neoplastic versus nonneoplastic pancreatic cystic lesions. Gastrointest Endosc 2003;58:59-64. doi:10.1067/mge.2003.298

15. Vilas-Boas F, Macedo G. Pancreatic Cystic Lesions: New Endoscopic Trends in Diagnosis. J Clin Gastroenterol 2018;52:13-19. doi:10.1097/ MCG.0000000000000945

16. Polkowski M, Jenssen C, Kaye P, et al. Technical aspects of endoscopic ultrasound (EUS)-guided sampling in gastroenterology: European 
Society of Gastrointestinal Endoscopy (ESGE) Technical Guideline March 2017. Endoscopy 2017;49:989-1006. doi:10.1055/s-0043-119219

17. Fusaroli P, Serrani M, De Giorgio R, et al. Contrast HarmonicEndoscopic Ultrasound Is Useful to Identify Neoplastic Features of Pancreatic Cysts (With Videos). Pancreas 2016;45:265-268. doi:10.1097/ MPA. 0000000000000441

18. Konda VJ, Aslanian HR, Wallace MB, Siddiqui UD, Hart J, Waxman I. First assessment of needle-based confocal laser endomicroscopy during EUS-FNA procedures of the pancreas (with videos). Gastrointest Endosc 2011;74:1049-1060. doi:10.1016/j.gie.2011.07.018

19. Nakai Y, Iwashita T, Park DH, Samarasena JB, Lee JG, Chang KJ. Diagnosis of pancreatic cysts: EUS-guided, through-the-needle confocal laser-induced endomicroscopy and cystoscopy trial: DETECT study. Gastrointest Endosc 2015;81:1204-1214. doi:10.1016/j. gie.2014.10.025

20. Napoleon B, Lemaistre AI, Pujol B, et al. A novel approach to the diagnosis of pancreatic serous cystadenoma: needle-based confocal laser endomicroscopy. Endoscopy 2015;47:26-32. doi:10.1055/s-0034-1390693

21. Keane MG, Wehnert N, Perez-Machado M, et al. A prospective trial of CONfocal endomicroscopy in CYSTic lesions of the pancreas: CONCYST-01. Endosc Int Open 2019;7:E1117-E1122. doi:10.1055/a-0957-2976

22. Fugazza A, Gaiani F, Carra MC, et al. Confocal Laser Endomicroscopy in Gastrointestinal and Pancreatobiliary Diseases: A Systematic Review and Meta-Analysis. Biomed Res Int 2016;2016:4638683. doi:10.1155/2016/4638683

23. Karia K, Kahaleh M. A Review of Probe-Based Confocal Laser Endomicroscopy for Pancreaticobiliary Disease. Clin Endosc 2016;49:462-466. doi:10.5946/ce.2016.086

24. Tanaka M, Fernandez-del Castillo C, Adsay V, et al. International consensus guidelines 2012 for the management of IPMN and MCN of the pancreas. Pancreatology 2012;12:183-197. doi:10.1016/j. pan.2012.04.004

25. Kohli DR, Kapoor A, BouHaidar D, Vachhani R. Requirement of a single high-risk feature as an indication for EUS for the diagnosis of asymptomatic pancreatic cysts. Pancreatology 2016;16:1015-1019. doi:10.1016/j.pan.2016.08.010

26. Brugge WR, Lewandrowski K, Lee-Lewandrowski E, et al. Diagnosis of pancreatic cystic neoplasms: a report of the cooperative pancreatic cyst study. Gastroenterology 2004;126:1330-1336. doi:10.1053/j. gastro.2004.02.013
27. Springer S, Wang Y, Dal Molin M, et al. A combination of molecular markers and clinical features improve the classification of pancreatic cysts. Gastroenterology 2015;149:1501-1510. doi:10.1053/j. gastro.2015.07.041

28. Singhi AD, McGrath K, Brand RE, et al. Preoperative next-generation sequencing of pancreatic cyst fluid is highly accurate in cyst classification and detection of advanced neoplasia. Gut 2018;67:21312141. doi:10.1136/gutjnl-2016-313586

29. Ngamruengphong S, Bartel MJ, Raimondo M. Cyst carcinoembryonic antigen in differentiating pancreatic cysts: a meta-analysis. Dig Liver Dis 2013;45:920-926. doi:10.1016/j.dld.2013.05.002

30. Valsangkar NP, Morales-Oyarvide V, Thayer SP, et al. 851 resected cystic tumors of the pancreas: a 33-year experience at the Massachusetts General Hospital. Surgery 2012;152(3 Suppl 1):S4-S12. doi:10.1016/j. surg.2012.05.033

31. Crippa S, Partelli S, Falconi M. Extent of surgical resections for intraductal papillary mucinous neoplasms. World J Gastrointest Surg 2010;2:347-351. doi:10.4240/wjgs.v2.i10.347

32. Kwong WT, Lawson RD, Hunt G, et al. Rapid Growth Rates of Suspected Pancreatic Cyst Branch Duct Intraductal Papillary Mucinous Neoplasms Predict Malignancy. Dig Dis Sci 2015;60:2800-2806. doi:10.1007/ s10620-015-3679-8

33. Kim KW, Park SH, Pyo J, et al. Imaging features to distinguish malignant and benign branch-duct type intraductal papillary mucinous neoplasms of the pancreas: a meta-analysis. Ann Surg 2014;259:72-81. doi:10.1097/ SLA.0b013e31829385f7

34. Crippa S, Bassi C, Salvia R, et al. Low progression of intraductal papillary mucinous neoplasms with worrisome features and high-risk stigmata undergoing non-operative management: a mid-term follow-up analysis. Gut 2017;66:495-506. doi:10.1136/gutjnl-2015-310162

35. Kwok K, Chang J, Duan L, Huang BZ, Wu BU. Competing Risks for Mortality in Patients With Asymptomatic Pancreatic Cystic Neoplasms: Implications for Clinical Management. Am J Gastroenterol 2017;112:1330-1336. doi:10.1038/ajg.2017.141

36. Crippa S, Pezzilli R, Bissolati M, et al. Active Surveillance Beyond 5 Years Is Required for Presumed Branch-Duct Intraductal Papillary Mucinous Neoplasms Undergoing Non-Operative Management. Am J Gastroenterol 2017;112:1153-1161. doi:10.1038/ajg.2017.43

37. Pergolini I, Sahora K, Ferrone CR, et al. Long-term Risk of Pancreatic Malignancy in Patients With Branch Duct Intraductal Papillary Mucinous Neoplasm in a Referral Center. Gastroenterology 2017;153:1284-1294.e1. doi:10.1053/j.gastro.2017.07.019 